\title{
Patterns of renal
}

\section{angiomyolipoma regression post embolisation on medium- to long-term follow-up}

\author{
K PATATAS, MB BS, FRCR, G J ROBINSON, BMBch, FRCR, D F ETTLES, MB ChB, FRCR and \\ R LAKSHMINARAYAN, MBBS, FRCR
}

Vascular Radiology Department, Hull Royal Infirmary, Hull, UK

Address correspondence to: Dr Kyriacos Patatas

E-mail:kpatatas@hotmail.com

Objective: To assess the patterns of regression of renal angiomyolipoma (AML) post embolisation and report the outcomes related to the use of different embolic materials.

Methods: A retrospective review of all patients who underwent embolisation for renal AML at our institution between January 2004 and April 2012.

Results: 13 patients underwent 16 episodes of embolisation. Coils were used as the primary embolisation material in 10 episodes and microspheres in 6 episodes. The size reduction rate highly correlated on CT followup between the two groups, with $25.6 \% \mathrm{vs}$ $22.7 \%$ reduction at 12 months, $27.5 \%$ vs $25.1 \%$ at 24 months, $35.0 \%$ vs $33.0 \%$ at 36 months and $35.0 \%$ vs $36.8 \%$ at 48 months. During follow-up, all tumours reduced in size with one patient requiring subsequent embolisation whose tumour reduced by only $6.5 \%$ after
1 year and subsequently exhibited regrowth after 4 years. Two patients presented with rebleeding and underwent repeat embolisation. Our overall retreatment rate (23\%) is well within the literature range (up to $37 \%$ ). None of the patients underwent surgery.

Conclusion: The majority of AML shrinkage occurs within the first year following embolisation and appears to plateau after 3 years, which could have an impact on follow-up strategy. The percentage reduction at 1 year may reflect the long-term effect of embolisation with tumours demonstrating minor size reduction more likely to relapse at long-term follow-up. Embolisation of renal AML produces durable long-term results regardless of the choice of embolic agent.

Advances in knowledge: These findings provide information to guide CT follow-up of renal $A M L$ post embolisation. 
Renal angiomyolipoma (AML) is a benign hamartomatous tumour that contains fat, smooth muscle and abnormal blood vessels in varying proportions [1]. There are two types of AML, a sporadic type that occurs predominantly in older patients and presents with a single unilateral lesion and a type associated with tuberous sclerosis (TS) in which lesions are multiple and bilateral. The sporadic type constitutes $80 \%$ of all cases [2].

The main complication of AML is retroperitoneal haemorrhage, which can be severe and life threatening [3]. The bleeding originates from the abnormal vascular component that represents the angiogenic portion of the tumour. The risk of bleeding is increased with increasing tumour size, with $4 \mathrm{~cm}$ generally the accepted cut-off size for intervention [4,5]. Symptomatic lesions of any size should also be treated [6].

Selective embolisation has been established as the treatment of choice for large or symptomatic AML, as it can preserve a significant proportion of renal tissue and renal function $[7,8]$.

The purpose of this study was to report the outcomes related to the use of different embolic materials and assess the patterns of regression of renal AML post embolisation.

\section{MATERIALS AND METHODS}

All consecutive cases that underwent renal AML embolisation at our institution over the period from January 2004 to April 2012 were identified retrospectively from the radiology department's electronic records. Angiographic and cross-sectional imaging and reports were retrieved and reviewed. The site, maximum size and extent of AML were assessed by review of CT examinations performed prior to selective embolisation and post-embolisation tumour size and percentage reduction were evaluated from follow-up CT. Tumour size was measured as the longest diameter on axial CT images. Conventional unenhanced and enhanced axial scans were obtained at different institutions with different equipment and protocols but 5-mm reconstructed section thickness in the axial plane was available for all examinations. The frequency and duration of follow-up CT varied with the radiologist taking on this responsibility. The patients' medical records were also examined to determine the indication for treatment, procedural details (e.g. type of embolic material and size) and complications. Repeat or further treatment and the symptom-free period were also ascertained from clinical follow-up letters. Procedures were classified as elective or emergency. Elective embolisation was performed as a planned admission on a routine interventional list. Indications for elective embolisation were tumours $>4 \mathrm{~cm}$, pain or previous history of haemorrhage. Emergency cases were patients presenting with sudden onset of pain and signs suggesting acute bleeding. All cases were diagnosed with contrast-enhanced CT of the abdomen and pelvis. All cases were performed by two experienced interventional radiologists. One typically performs embolisation with coils (Nester ${ }^{\circledR}$ Microcoils; Cook Medical, Bloomington, IN) to the inflow artery as the primary embolic agent, with infrequent and minimal use of particles to the tumour circulation (either microspheres or polyvinyl alcohol). The other uses 500- to $700-\mu \mathrm{m}$ microsphere particles (Embosphere ${ }^{\mathrm{TM}}$ Microspheres; Merit Medical, Salt Lake City, UT) as the primary embolic agent to occlude the tumour circulation. The end point of embolisation was stasis within the tumour circulation (Figure 1).

\section{RESULTS}

13 patients underwent 16 episodes of embolisation for 13 tumours. The mean age was 59 years. Only 2 patients $(2 / 13,15 \%)$ had TS. 12 cases were performed electively, 11 for large $(>4 \mathrm{~cm}$ ) lesions (4 of which were also causing recurrent pain) and 1 for previous bleeding. Four emergency embolisation procedures were performed for acute retroperitoneal bleeding. All patients underwent $\mathrm{CT}$ prior to the procedure. The mean AML size was $6.2 \mathrm{~cm}$ (range $4.2-10.0 \mathrm{~cm}$ ).

Coils were used as the primary embolisation material in 10 episodes (Group 1) and microspheres in 6 episodes (Group 2). Immediate primary technical success was achieved in all 13 patients (100\%). Tumour rupture with contrast extravasation was observed in only 1 procedure $(1 / 14,7 \%)$ during selective embolisation using coils as a primary embolic agent. This was treated with coil occlusion of the inflow upper pole renal artery. Other complications included two cases (14\%) of post-embolisation syndrome (PES) and one case (7\%) of non-target embolisation, all of which were managed conservatively. In the latter case, CT of the abdomen 3 months after the procedure demonstrated a minor area of infarction in the posteromedial mid-renal level. PES was defined as flank pain, usually accompanied by fever, nausea and vomiting. Typical symptoms associated with 
Figure 1. Digital subtraction angiogram prior to embolisation ( $a$ and b) shows hypervascular tumour with a single feeder vessel at the upper pole of the right kidney. (c, d) Marked reduction in vascularity post-selective embolisation with embosphere particles and microcoils.
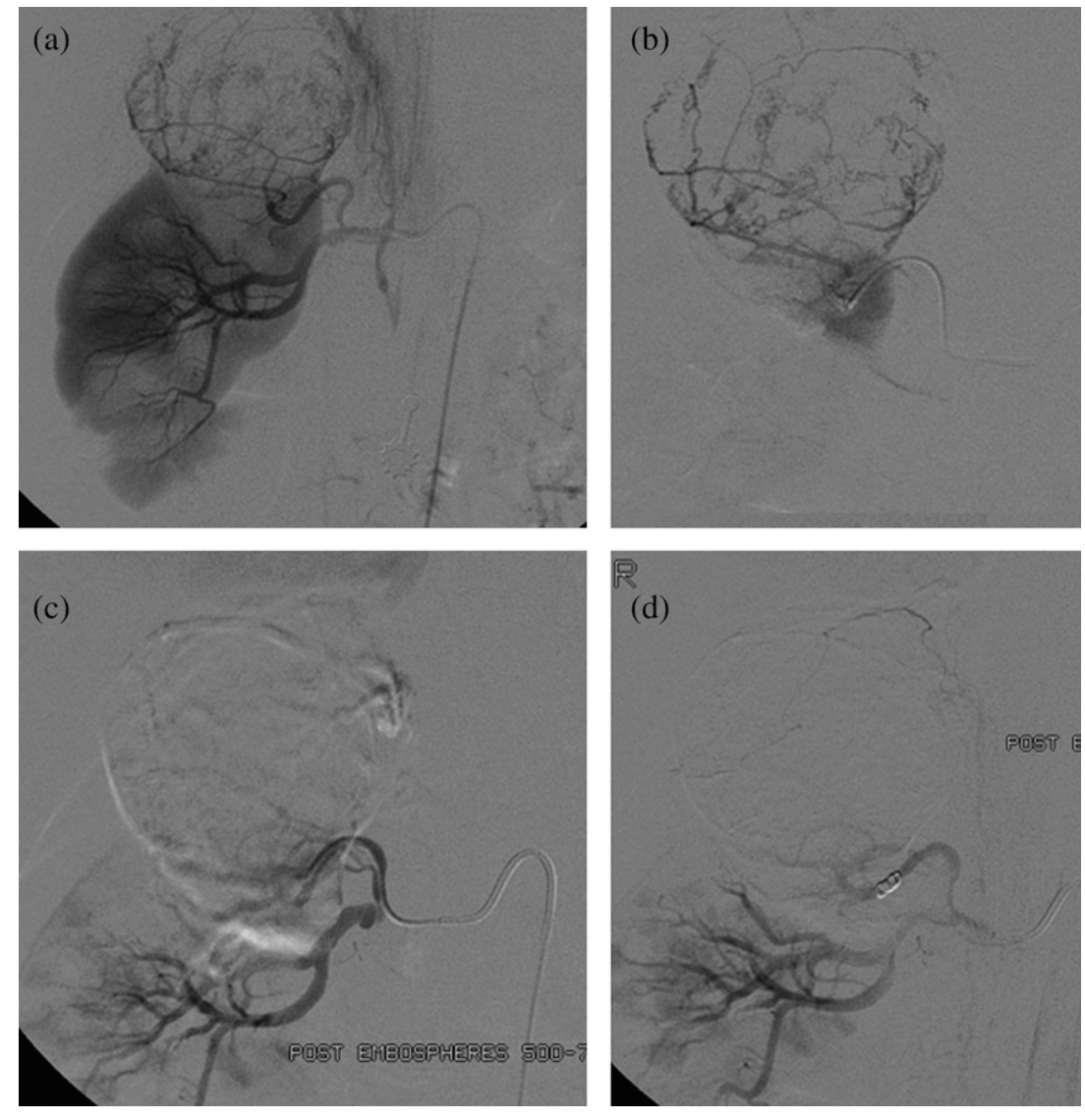

PES typically begin in the first $24 \mathrm{~h}$ post procedure and cease on average after $72 \mathrm{~h}$, although there can be significant variation in the time scale.

Serial CT was used for follow-up in all patients. Followup CT scans obtained 9-92 months after treatment were available for all patients. The size reduction rate highly correlated on follow-up between the two groups, with $25.6 \%$ vs $22.7 \%$ reduction at 12 months, $27.5 \%$ vs $25.1 \%$ at 24 months, $35.0 \%$ vs $33.0 \%$ at 36 months and $35.0 \%$ vs $36.8 \%$ at 48 months (Figure 2). $80 \%$ of tumours followed this pattern of regression. The overall reduction rate was $6.5 \%$ at 3 months, $16.0 \%$ at 6 months, $24.0 \%$ at 12 months, $26.5 \%$ at 2 years, $33.0 \%$ at 3 years and $36.0 \%$ at 4 years. During follow-up, all tumours reduced in size, with only one patient $(8 \%)$ requiring subsequent elective embolisation whose tumour reduced by only $6.5 \%$ after 1 year and subsequently exhibited regrowth after 4 years. Two patients (15\%) presented with rebleeding and underwent repeat embolisation as emergency cases. One had TS and presented 6 years after the previous embolisation procedure, and the other presented prior to the 1-year follow-up scan but with a satisfactory 3-month CT that had showed $8 \%$ size reduction of the AML size. 10 patients $(10 / 13,77 \%)$ remained symptom-free after a median follow-up period of 46 months (range 6-92 months). None of the patients underwent surgery.

\section{DISCUSSION}

Renal AMLs are benign tumours that consist of varying proportions of fat, smooth muscle and blood vessels. They are clinically important owing to their tendency to bleed. Approximately $60 \%$ of patients with large AMLs will present with a combination of acute bleeding, flank pain, haematuria and tender mass [1]. The blood vessels within AMLs are abnormal with no internal elastic lamina, tortuous and prone to aneurysmal formation and rupture [9]. Intervention is generally advised for symptomatic tumours or tumours that are $>4 \mathrm{~cm}$. Treatment options include embolisation and surgery. Embolisation has a major advantage over surgery of 
Figure 2. Pattern of percentage reduction ( $y$-axis) in the size of renal angiomyolipomas on CT follow-up post embolisation ( $x$-axis) demonstrates high correlation between the two groups (coils were used as the primary embolic agent in Group 1 and embosphere particles in Group 2).

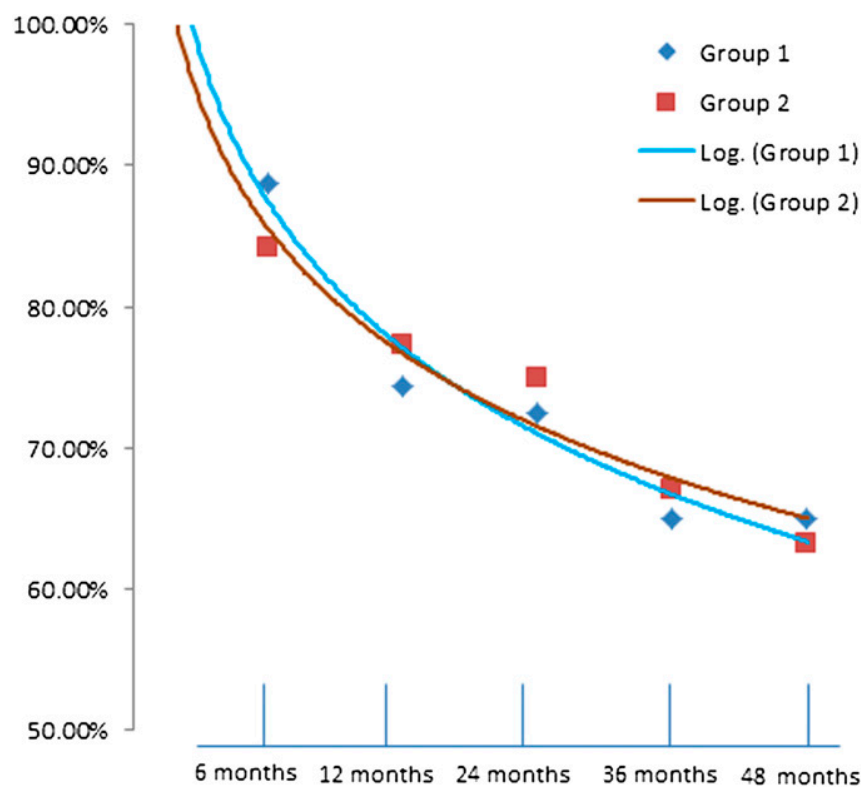

sparing a greater proportion of functioning renal tissue. However, the long-term results of the procedure are limited and there is little information in the literature regarding frequency and length of follow-up.

There is also no clear consensus in the literature regarding the embolisation technique and the choice of embolic agent [5]. Liquid embolic agent or particles $[4,7,8,10]$, coils in the inflow artery [7] and a combination of occlusion of the distal vascular bed with particles followed by coil occlusion of the arterial inflow [9] to prevent retrograde filling of the aneurysm have been described. The use of only coils in the embolisation of AML has been the subject of debate, with opponents of this technique stating that collaterals can form at the level of occlusion and make further embolisation difficult or impossible [4]. However, other investigators [9] believe that as AMLs are tumours with a vascular component they behave like, and must be treated as, part tumour and part aneurysm. This means that the source vessel requires occlusion along with the tumour circulation, with coil placement being the most efficient technique. They have demonstrated that, even though using particles alone may angiographically achieve stasis, the vascular tumour can remain pressurised and thus at risk of rupture.
Figure 3. Suggested algorithm for follow-up post embolisation.

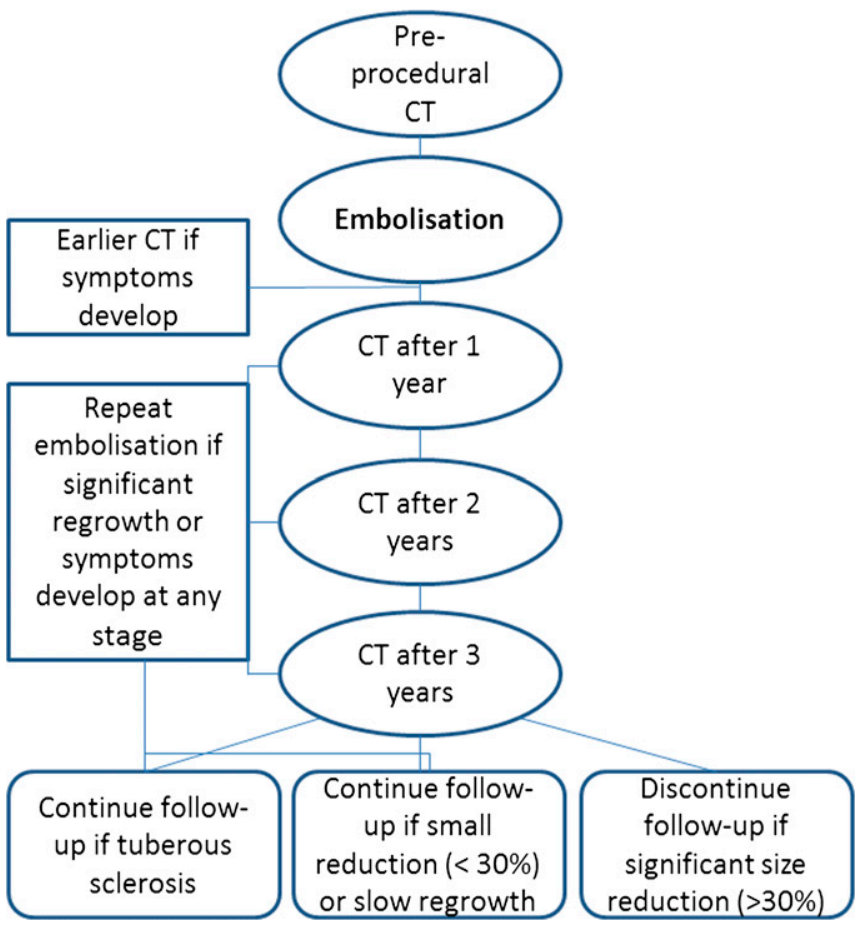

In the present study, we have demonstrated that embolisation is an effective long-term treatment for large or symptomatic AMLs and the outcomes of different embolisation techniques are comparable. Elective repeat embolisation was required in only 1 out of all patients $(1 / 13,8 \%)$, in whom particles were used as the primary and sole embolic agent. Two further patients presented with rebleeding and underwent repeat embolisation, one from each group. Our overall retreatment rate $(3 / 13,23 \%)$ is well within the reported literature range of up to $37 \%[4,7-11]$. $77 \%$ of patients remain symptom-free and intervention-free after a median follow-up period of almost 4 years.

Rupture with contrast extravasation was observed in only 1 out of 14 procedures (7\%) after embolisation with coils and this again correlates with other published series, with a maximum rupture rate of $30 \%$ reported by Lenton et al [9]. Embolisation in the study by Lenton et al was performed with a combination of 350 - to 500- $\mu \mathrm{m}$ polyvinyl alcohol (PVA) particles to occlude the distal vascular bed followed by coils to block the arterial inflow and prevent retrograde filling of the aneurysm. Ewalt et al [7], on the other hand, reported the embolisation of 27 AMLs using PVA and coils in the feeding artery and reported no intraprocedural rupture. 
Figure 4. Contrast-enhanced axial CT shows predominantly fatty angiomyolipoma before embolisation (a), which exhibited modest reduction in size 1 year post embolisation (b).

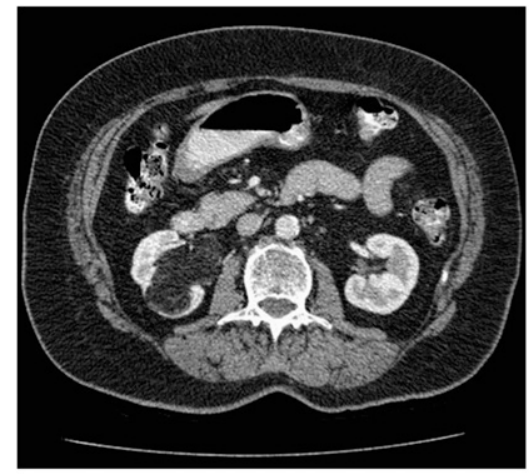

(a)

The small number of patients does not allow statistical analysis; however, size reduction rates were comparable between the two groups, as demonstrated in Figure 2, and maximum shrinkage (mean of $24 \%$ ) was observed by 1 year with continued reduction demonstrated up to 3 years. We observed a mean size reduction of $36 \%$ at 4 years, which is comparable with a mean reduction rate that varied from $26 \%$ to $43 \%$, as reported by other investigators $[2,4,11,12]$. However, the reduction rate at 36 months differed little from that at 48 months, which could imply the rate reaching a plateau. This could have an impact on the follow-up approach. In addition, a small or no reduction was demonstrated after 3 months (mean $6.5 \%$ ), which suggests that early CT is not beneficial unless there is occurrence of symptoms suggesting complication or regrowth. One possible regime for follow-up of embolised AML (Figure 3) would be to repeat CT yearly for 3 years, with discontinuation of follow-up if significant size reduction has occurred during 3 years with no evidence of regrowth. If significant regrowth is demonstrated at any of the follow-up scans, more frequent

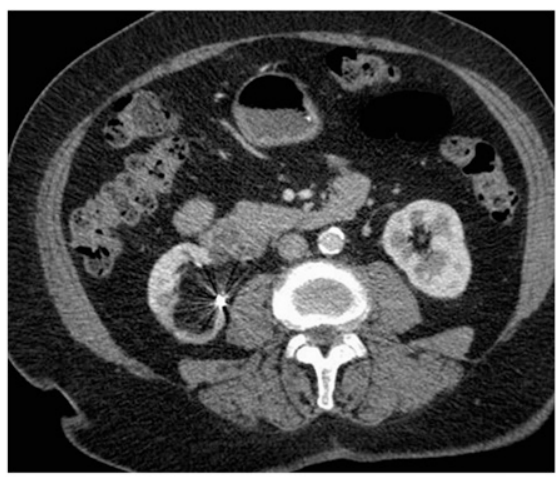

(b)

imaging may be required. If minor reduction is observed after 3 years, continued follow-up would be indicated. We consider the reduction rate at 1 year to reflect the longterm effect of embolisation, with tumours demonstrating minor size reduction being more likely to relapse at longterm follow-up. The AML that required repeat embolisation in our series reduced only $6.5 \%$ after 1 year and subsequently exhibited regrowth after 4 years. Lee et al [13] also recently showed mean AML size reduction of $33 \%$ and $43 \%$ at short-term ( $<6$ months) and long-term (>6 months) follow-up, and that tumour shrinkage occurring within 6 months after the procedure may reflect the long-term effects of embolisation. As suggested by Kothary et al [4] patients with TS may behave differently and require lifelong follow-up given the propensity for recurrence and multifocal disease. In our series, one patient with TS presented with rebleed 6 years after successful embolisation.

It is important to emphasise that-as noted by previous investigators [10] — the more clinically significant

Figure 5. Contrast-enhanced axial CT shows angiomyolipoma with a significant angiomyogenic component (a), which exhibited a marked reduction in size 2 years post embolisation with residual tumour mainly composed of fat (b).

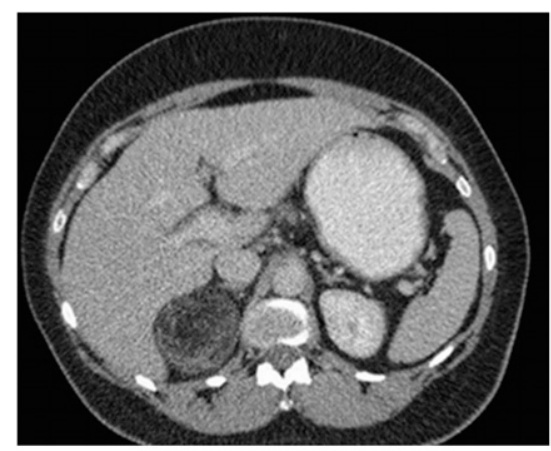

(a)

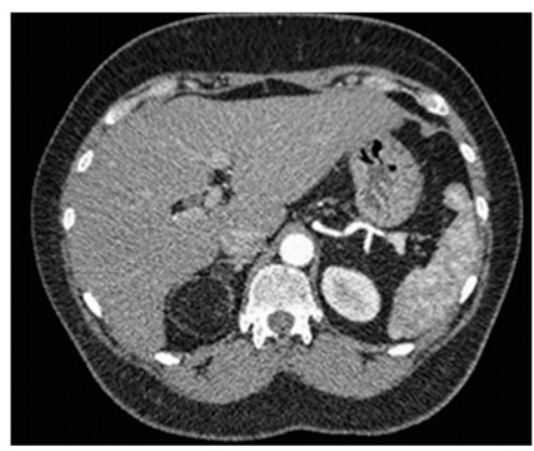

(b) 
angiomyogenic portion of the tumour appears to reduce in size disproportionally compared with the fatty component, which explains the variability in overall reduction of the AML sizes. The fat component appears to be relatively insensitive to the effect of embolisation (Figure 4), whereas the vascular component responds well (Figure 5). Therefore, the post-treatment size reduction does not always correlate with the success of the procedure [5].
In conclusion, the majority of AML shrinkage occurs within the first year following embolisation and appears to plateau after 3 years, which could have an impact on the follow-up approach. The embolisation of renal AML produces durable long-term results with a low rate of tumour relapse, and a considerable size reduction pattern was observed regardless of the choice of embolic agent.

\section{REFERENCES}

1. Nelson CP, Sanda MG. Contemporary diagnosis and management of renal angiomyolipoma. J Urol 2002;168:1315-25. doi: 10.1097/01. ju.0000028200.86216.b2.

2. Rimon U, Duvdevani M, Garniek A, Golan G, Bensaid P, Ramon J, et al. Ethanol and polyvinyl alcohol mixture for transcatheter embolization of renal

angiomyolipoma. AJR Am J

Roentgenol 2006;187:762-8. doi: 10.2214/AJR.05.0629.

3. Soulen MC, Faykus MH Jr, Shlansky-Goldberg RD, Wein AJ, Cope C. Elective embolization for prevention of haemorrhage from renal angiomyolipoma. J Vasc Interv Radiol 1994;5:587-91.

4. Kothary N, Soulen MC, Clark TW, Wein AJ, Shansky-Goldberg RD, Crino PB, et al. Renal angiomyolipoma: long-term results after arterial embolisation. J Vasc Interv Radiol 2005;16:45-50. doi: $10.1097 / 01$. RVI.0000143769.79774.70.

5. Halpenny D, Snow A, McNeill G, Torreggiani WC. The radiological diagnosis and treatment of renal angiomyolipoma-current status. Clin Radiol 2010;65:99-108. doi: 10.1016/j.crad.2009.09.014.

6. Mourikis D, Chatzioannou A, Antoniou A, Kehagias D, Gikas D, Vlahos L. Selective arterial embolization in the management of symptomatic renal angiomyolipomas. Eur J Radiol 1999;32: 153-9.

7. Ewalt D, Diamond N, Rees C, Sparagana SP, Delgado M, Batchelor L, et al. Long-term outcome of transcatheter embolisation of renal angiomyolipomas due to a tuberous sclerosis complex. J Urol 2005; 174:1764-6. doi: 10.1097/01. ju.0000177497.31986.64.

8. Lee W, Kim T, Chung JW, Han JK, Kim SH, Park JH. Renal angiomyolipoma: embolotherapy with a mixture of alcohol and iodized oil. J Vasc Interv Radiol 1998;9: 255-61.

9. Lenton J, Kessel D, Watkinson AF. Embolization of renal angiomyolipoma: immediate complications and long-term outcomes. Clin Radiol 2008;63:864-70. doi: 10.1016/j.crad.2008.02.005.
10. Han YM, Kim JK, Roh BS, Song HY, Lee JM, Lee YH, et al. Renal angiomyolipoma: selective arterial embolization-effectiveness and changes in angiomyogenic components in long term follow up. Radiology 1997;204:65-70.

11. Ramon J, Rimon U, Garniek A, Golan G, Bensaid P, Kitrey ND, et al. Renal angiomyolipoma: longterm results following selective arterial embolisation. Eur Urol 2009;55:1155-62. doi: 10.1016/j. eururo.2008.04.025.

12. Harabayashi T, Shinohara N, Katano H, Nonomura K, Shimizu T, Koyanagi T. Management of renal angiomyolipomas associated with tuberous sclerosis complex. J Urol 2004;171:102-5. doi: 10.1097/01.ju.0000100100. 36354.61 .

13. Lee SY, Hsu HH, Chen YC, Huang CC, Wong YC, Wang LJ, et al. Embolisation of renal angiomyolipomas: short-term and long-term outcomes, complications and tumour shrinkage. Cardiovasc Intervent Radiol 2009;32:1171-8. doi: 10.1007/s00270-009-9637-0. 